Erschienen in: Stickel, Gerhard (Hrsg.): Sprache - Sprachwissenschaft - Öffentlichkeit.

Berlin/New York: de Gruyter, 1999. S. 243-261.

(Jahrbuch des Instituts für Deutsche Sprache 1998)

DOI: $h$ ttps://doi.org/10.1515/9783110622645-016

\title{
LUDWIG JÄGER
}

\section{Linguistik und Öffentlichkeit Aspekte eines schwierigen Verhältnisses}

\begin{abstract}
Der Vortrag geht aus von dem weithin geteilten Befund, daß die Sprachwissenschaft sowohl in der Öffentlichkeit als auch im Wissenschaftsdiskurs der Kulturwissenschaften an Ansehen verloren hat. Er versucht vor dem Hintergrund dieses Befundes das Verhältnis von Linguistik und Öffentlichkeit zu erörtern. Die Resonanzlosigkeit der Sprachwissenschaft im öffentlichen Raum wird zunächst im Kontext der These diskutiert, die Disziplin befolge nicht die „Gesetzmäßigkeiten der Darstellung" (Münch) und verfüge über kein angemessenes Marketing. Eine solche Ursachenzuschreibung für die mangelnde Öffentlichkeitsfähigkeit linguistischen Wissens wird als zu kurz greifend zurückgewiesen. Die Ansehenskrise wird statt dessen in einer grundsätzlicheren Hinsicht zurückgeführt (1) auf die allgemeinere Krise des wissenschaftlichen Wissens in der „Expertengesellschaft“ (Hitzler) sowie (2) auf eine für die Sprachwissenschaft charakteristische mangelnde Zeitgenossenschaft. Diese ist - so die These des Vortrags - herzuleiten aus einem geisteswissenschaftlichen Autonomieverständnis, das einer Verschränkung von Wissenschaft und öffentlich-politischem Handeln kritisch gegenübersteht sowie im besonderen aus der stummen Distanz der Sprachwissenschaft zu dem - von der Öffentlichkeit mit großer Aufmerksamkeit verfolgten - Mediendiskurs der Kulturwissenschaften.
\end{abstract}

\section{Klage über die Außenansicht: Die öffentliche Resonanzlosigkeit der Sprachwissenschaft}

Nicht zuletzt das Thema dieser Jahrestagung des ,Instituts für Deutsche Sprache" zeigt, daß die germanistische Linguistik begonnen hat, das Problem der Außenansicht zu reflektieren, die sie dem bildungs- und wissenschaftspolitischen System sowie der breiteren Öffentlichkeit der Bundesrepublik gegenwärtig darbietet. Allenthalben stoßen wir in den letzten Jahren auf Versuche, sich dem Umstand zu stellen, daß die Linguistik, nachdem sie in den sechziger Jahren, der Phase ihrer strukturalistischen Blüte, kurz im Verdacht stand, eine Begründungswissenschaft der Geisteswissenschaften zu werden', aus der Perspektive ihrer Außenwahrnehmung

I Vgl. Böhme/Scherpe 1996, S. 10. 
in öffentliche Bedeutungslosigkeit versunken ist. So stellten etwa die „Mitteilungen des Deutschen Germanistenverbandes“ das Heft 3 des Jahres 1994 unter das Leitthema „Die Germanistik und die Öffentlichkeit". In seinem Editorial beklagte Hans-Jürgen Bachorsky u. a., daß es ,mit dem öffentlichen Ansehen der Germanistik, immerhin des größten geisteswissenschaftlichen Faches, [...] nicht allzu weit her zu sein" scheine. „Nicht nur Politik und Verwaltung“ seien sich - so heißt es hier - „einig, daß dieses Fach und seine Leistungen in Forschung und Lehre von so geringem Wert" seien, „daß man an ihm immer neue Sparprogramme exekutieren“ könne. Auch von den Medien fühle es sich „stiefmütterlich behandelt" und entsprechend schwach ausgebildet sei insofern auch sein Selbstbewußtsein. ${ }^{2}$ Rainer Wimmer stellte am gleichen Ort die skeptische Frage, ob es für die germanistische Linguistik überhaupt noch eine interessierte Öffentlichkeit gebe und stimmte mit Rudolf Hoberg in dem Befund überein, daß das, was „Germanisten in ihrer Wissenschaft so alles treiben, [...] die Öffentlichkeit kaum" mehr beschäftige. ${ }^{3}$ In einem kleinen Aufsatz zum Thema "Öffentlichkeit und Sprachwissenschaft" hat Rudolf Hoberg jüngst seine These weiter pointiert und darüber Klage geführt, daß zwar das „Interesse an der Sprache im allgemeinen, an der deutschen Sprache im besonderen, auch an bestimmten Varietäten - etwa den Fachsprachen oder der Jugendsprache -, auch an einzelnen sprachlichen Erscheinungen“" in vielen Wissenschaften, aber auch in anderen Bereichen sehr groß sei, man jedoch von einem „Bestreben, linguistische Erkenntnisse aufzunehmen und mit Sprachwissenschaftlern in einen Dialog zu treten", kaum noch etwas spüren könne. ${ }^{4}$

Das erste Resümee zu diesem Befund formulierte dann Gerd Antos in seinem 1996 erschienenen Buch „Laien-Linguistik“, wo es am Ende des ersten Kapitels über das ,publizistische Erscheinungsbild der Linguistik in der Öffentlichkeit" heißt:

„Es führt kaum ein Weg an der Erkenntnis vorbei, daß die Linguistik trotz aller beeindruckenden Erfolge zumindest im primären Wissenschaftssektor - eine Wissenschaft ohne ,Verbraucher" [...], ohne quantitativ bedeutsame Berufsfelder, ohne Publizität, ohne einen ausreichenden Markt für populärwissenschaftliche Verbreitung, kurz: ohne eine tiefgehende Verankerung in der Öffentlichkeit ist."

Wie auch immer man zu der Diagnose von Bachorsky, Wimmer, Hoberg und Antos steht, richtig scheint zu sein - und dies vermag nicht zuletzt die rhetorische und inhaltliche Form der in den Medien ausgetrage-

\footnotetext{
Vgl. Bachorsky 1994, S. 10-15.

Vgl. Wimmer 1994, S. 51-56.

Vgl. Hoberg 1997, S. 54.

Antos 1996, S. 12.
} 
nen Debatte über die Rechtschreibreform zu verdeutlichen -, daß mit der öffentlichen Wertschätzung der (germanistischen) Sprachwissenschaft etwas nicht in Ordnung ist: Ihre Außenansicht ist insofern problematisch geworden, als das Fach sowohl im Wissenschaftsdiskurs der Kulturwissenschaften als auch in der Öffentlichkeit - vielleicht glücklicherweise noch nicht in gleichem Maße im politischen System - entschieden an Ansehen verloren hat. Angesichts eines sich verschärfenden Verteilungskampfes um knapper werdende Ressourcen und angesichts steigender Legitimitätsanforderungen bezüglich - um Rainer Wimmer noch einmal zu zitieren - „des Geldes, das man verbraucht, oder bezüglich des Einflusses, den man nimmt (etwa auf den Schulunterricht), oder bezüglich der Aufmerksamkeit, die man erheischt, oder der Ratschläge, die man gibt" ${ }^{\text {"6 }}$, ist es also sicher begrüßenswert, daß die germanistische Sprachwissenschaft sich ihrer Resonanzlosigkeit bewußt wird und über die Möglichkeit der Veränderung dieses bedrohlichen Zustandes der Disziplin nachsinnt.

Wer nun allerdings erwartet hätte, daß sich das Fach angesichts der durchaus konsensfähigen Diagnose in therapeutischer Absicht auf den Weg einer disziplinären Standortbestimmung begeben würde, sieht sich bei näherer Betrachtung der noch jungen Debatte über das Problem der Resonanzlosigkeit eines anderen belehrt. Es hat bislang nicht den Anschein - vielleicht vermochte diese Tagung das Bild zu korrigieren -, als ließe sich die Entdeckung des schwierigen Verhältnisses von Sprachwissenschaft und Öffentlichkeit als der Beginn einer Reflexion des ohne Zweifel zutreffenden Befundes werten, daß wir es mit einer Krise der Öffentlichkeitsfähigkeit sprachwissenschaftlichen Wissens zu tun haben, einer Krise, an der die Sprachwissenschaft selbst ja nicht ganz unbeteiligt sein kann. Durchaus überraschend ist deshalb die Richtung, die die über den Befund hinausgehende Analyse des diagnostizierten Übels nimmt. In den Versuch einer Ursachenbestimmung scheint sich nämlich in den bisherigen Beiträgen zum Thema eine Rhetorik der Klage einzuschleichen, der Klage über die - so Hoberg - ,mangelnde Dialogbereitschaft in den anderen Wissenschaften, den Medien und im Alltag " ${ }^{\text {, }}$, bzw. die Klage so Antos - über „die Rezeptionsverweigerung der Öffentlichkeit gegenüber Erkenntnissen der modernen Linguistik. ${ }^{68}$

Natürlich ist es, wenn man die Schwierigkeiten im Verhältnis von Sprachwissenschaft und Öffentlichkeit vor allem auf die Verweigerung des (von Linguisten gesuchten?) Dialogs durch potentielle fachexterne Dialogpartner bzw. auf deren Nicht-Zurkenntnisnahme linguistischen Wissens zurückführt, durchaus konsequent, daß die bisher gemachten Thera-

6 Wimmer 1994, S. 51.

7 Hoberg 1997, S. 59.

8 Antos 1996, S. 3. 
pievorschläge nicht etwa auf eine Veränderung des Produkts Sprachwissenschaft, sondern vor allem auf die Verbesserung des werblichen Auftritts der Disziplin auf dem Markt abzuzielen scheinen. Wenn die Adressaten durch gutgemeinte Kommunikationsversuche nicht erreicht werden und wenn Zweifel an der Qualität des Angebots nicht bestehen, empfiehlt es sich, über die kommunikative Strategie nachzudenken, will man den Rezipienten nicht Mutwilligkeit oder kognitive Beschränkung unterstellen. Und tatsächlich darf ja auch eine der Ursachen für das gegenwärtig gestörte Verhältnis von Sprachwissenschaft und Öffentlichkeit durchaus in einer unbefriedigenden externen Kommunikation der Disziplin mit der Öffentlichkeit gesehen werden. Rainer Wimmer ist insofern zuzustimmen, wenn er diagnostiziert:

„Die Schwerverständlichkeit wissenschaftsorientierten Redens und Schreibens, unzureichende Erfahrungen mit Vermittlungssituationen und -praktiken sowie ein Mangel an guten populärwissenschaftlichen Texten“" seien ,die wesentlichen Ursachen für die unzureichende Resonanz der (Sprach)germanistik in den Medien und in der außer-bzw. überfachlichen Öffentlichkeit." “9

Vergegenwärtigt man sich nämlich die „Kommunikationszwänge“, die - wie der Soziologe Richard Münch jüngst gezeigt hat - moderne Kommunikationsgesellschaften bestimmen, mutet das kommunikative Verhalten der (germanistischen) Sprachwissenschaft in der Öffentlichkeit - sieht man einmal von dem verdienstvollen 'Sprachreport' des IDS ab - tatsächlich weithin anachronistisch an. Kommunikation werde - so Münch „zum strategischen Spiel, das über Erfolg und Mißerfolg von Individuen, Organisationen, gesellschaftlichen Gruppen und ganzen Gesellschaften" entscheide. Die Lebensverhältnisse der gesellschaftlichen Klassen, Schichten und Gruppen seien davon abhängig, wie gut sie sich in Szene zu setzen bzw. wie lautstark sie sich im öffentlichen Diskurs zu Worte zu melden vermöchten. Dies gelte im übrigen gerade auch für die kulturellen gesellschaftlichen Subsysteme:

„Literatur, bildende Kunst, Musik, aber auch Religion und Wissenschaft sind heute so weit in den öffentlichen Kommunikationsprozeß eingeflochten, daß sie den Gesetzmäßigkeiten der Darstellung unterworfen werden. Ihr Erfolg und ihre Verbreitung hängt von einem geschickten Marketing ab."10

Folgte man also der skizzierten Argumentation, dann wäre das Problem der öffentlichen Resonanzlosigkeit der Sprachwissenschaft bzw. - wie im Falle der Rechtschreibreform - das der selektiv aggressiven Resonanz weniger ein Problem der disziplinären Verfassung des Faches, als vielmehr in erster Linie eines seiner kommunikativen Ungeschicklichkeit gegen-

9 Wimmer 1994, S. 52.

$10 \mathrm{Vgl}$. Münch 1995, S. $82 \mathrm{f}$. 
über einer sich aufgrund dieser Ungeschicklichkeit verweigernden Öffentlichkeit. Die Schwierigkeiten des Faches wären also weniger innerdisziplinärer Natur als vielmehr Ausdruck eines schlechten Marketings. Die Nicht-Öffentlichkeitsfähigkeit des linguistisch-disziplinären Wissens hätte seine Ursachen auch nicht in Merkmalen des Wissens selbst, sondern in der unvollkommenen Beherrschung der „Künste seiner Darstellung. "11 So richtig nun die Konstatierung eines Mangels an angemessener Darstellung des Faches in der Öffentlichkeit auch sein mag, alle relevanten Gründe für die Krise der Öffentlichkeitsfähigkeit linguistischen Wissens bzw. für das Verschwinden einer an das Fach gerichteten Relevanzerwartung der Öffentlichkeit und des politischen Systems sind damit nicht benannt.

Erlauben Sie mir hier eine Zwischenbemerkung: Das Kriterium der Öffentlichkeitsfähigkeit linguistischen Wissens ist natürlich an sich nicht notwendigerweise ein Gütekriterium für die wissenschaftliche Qualität dieses Wissens. Ohne Zweifel gibt es viele Bereiche unverzichtbarer und qualitätvoller linguistischer Forschung, die - etwa aufgrund der formalen Natur ihrer Metasprachen oder aufgrund der Komplexität ihrer Modellbildungen - vorderhand nicht dazu prädestiniert sind, jenseits des Wissenschaftssystems öffentlich geschätzt zu werden. Gleichwohl wird es immer mehr zu einem Qualitätskriterium jeglicher Wissenschaft werden, daß es den Vertretern einer Disziplin gelingt, die Notwendigkeit ihrer Forschungen externen Adressaten - also etwa der Laienöffentlichkeit oder den Vertretern öffentlicher Geldgeber - in deren Relevanzhorizont zu vermitteln. Ein eindrucksvolles Beispiel dafür, wie etwa die Notwendigkeit differenzierter grammatischer Analyse für die Kritik an laien-linguistischer Sprachkritik in erhellender Weise vermittelt werden könnte, hat uns Peter Eisenberg gegeben.

Ich möchte nun im folgenden versuchen, einige der - wie mir scheint - über das Marketing-Problem hinausgehenden Gründe für die Krise der Öffentlichkeitsfähigkeit linguistischen Wissens zu skizzieren und werde dabei zunächst einen Blick auf kommunikative Vermittlungsprobleme des Wissenschaftssystems insgesamt werfen.

\section{Der Aufstand der Laien und das „postmoderne Wissen“}

Jean-François Lyotard hat in seinem berühmten Essay von 1979 über ,Das postmoderne Wissen"12 den Zustand der Postmoderne als den einer Kultur gekennzeichnet, deren Subsysteme Literatur, Kunst und Wissenschaft

11 Münch 1995, S. 89.

12 Lyotard 1986. 
Ende des 19. Jahrhunderts grundlegenden Transformationen unterworfen worden seien. ${ }^{13}$ Diese Transformationen lassen sich für ihn durch eine Reihe von Leitvokabeln charakterisieren, die vor allem um zwei Begriffe gruppiert sind: um die Begriffe Dissemination und Delegitimierung. Mit Dissemination kennzeichnet Lyotard die Zerstreuung der postmodernen Kultur, die für ihn eine Folge ihres Legitimationsverlustes, also eine Folge der generellen Delegitimierung überkommener Formen kognitiver Orientierung darstellt. Während Prämoderne und Moderne jeweils über eine so Lyotard - Erzählung verfügten, aus der sich die Legitimität des Wissens und damit auch die Legitimität von Gesellschaften habe herleiten lassen, gelte dies für die postmoderne Kultur nicht mehr:

„In der gegenwärtigen Gesellschaft und Kultur, also in der postindustriellen Gesellschaft, der postmodernen Kultur, stellt sich die Frage der Legitimierung des Wissens in anderer Weise. Die große Erzählung hat ihre Glaubwürdigkeit verloren [...]."14

Eine postmoderne Kultur ist also eine Kultur, die nicht mehr - wie die Prämoderne und die Moderne - über Verfahren der einheitsstiftenden ,Selbstrechtfertigung ${ }^{\text {"15 }}$ in Religion, Philosophie und Wissenschaft verfügt und die deshalb in vielen Hinsichten ,zerstreut" und ,zersplittert" ${ }^{\text {"16 }}$ ist.

Die aus dem Legitimationsverlust entspringende Zerstreuung gilt (1) einerseits für das ,,soziale Band der Gesellschaften"17, das - wie Lyotard formuliert - nicht mehr ,aus einer einzigen Faser gemacht ist“ und dessen Zerfaserung deshalb zu einer Auflösung des ,sozialen Subjektes“18 führt und sie gilt (2) andererseits für die verlorene identitätsverbürgende Funktion von Philosophie und Wissenschaften sowie für deren Sprachspiele. Sie betrifft somit insgesamt den Status dessen, was wir Wissen nennen:

„Die Krise des wissenschaftlichen Wissens, deren Anzeichen sich bereits seit dem Ende des 19. Jahrhunderts vervielfachen, entstammt nicht einer zufälligen Wucherung der Wissenschaften, die selbst die Wirkung des Fortschrittes der Techniken und der Ausbreitung des Kapitalismus wäre. Sie ergibt sich aus der inneren Erosion des Prinzips der Legitimität des Wissens." 19

\footnotetext{
Vgl. Lyotard 1986, S. 13.

Lyotard 1986, S. 112.

Lyotard 1986, S. $30 \mathrm{ff}$.

Vgl. Lyotard 1986, S. $119 \mathrm{ff}$.

Lyotard 1986, S. 42 ff.

Vgl. Lyotard 1986, S. 119.

19 Lyotard 1986, S. 116.
} 
Während die Prämoderne die Legitimität des Wissens aus der mythischen Erzählung und die Moderne aus der Rationalität der wissenschaftlichen Rede bezog, ist für die Postmoderne - so Lyotard - die Idee der einheitsstiftenden Metasprache zerbrochen. Kennzeichnend für sie ist das Modell einer nicht mehr durch Metainstanzen zu steuernden Diskursvielfalt, d.h. das Modell eines durch Diskursregeln nicht mehr zu schlichtenden Streites. ${ }^{20}$ Auch die Diskurse der Wissenschaften folgen deshalb der allgemeinen Maxime, „daß Sprechen Kämpfen im Sinne des Spielens ist und daß Sprechakte einer allgemeinen Agnostik angehören." ${ }^{\text {21 }}$

Postmodernes Wissen ist also die spezifische Gestalt, die das Wissen unter den Bedingungen einer Kultur annimmt, die - angesichts der „Krise der modernen Ideale" ${ }^{\text {"22 } 2}$ - die sichere Behausung der Rechtfertigungssysteme und der ihnen zugeordneten Diskurse verlassen hat. Obsolet geworden in der Postmoderne sind deshalb - so Lyotard - die großen philosophischen und theoretischen Leitideen, die kantische Vernunft der Aufklärer ebenso wie der Hegelsche absolute Geist, das Marxsche Kollektivsubjekt der Arbeiterklasse genauso wie der Saussuresche Strukturalismus oder die selbstreferentiellen Systeme Luhmanns wie schließlich auch die herrschaftsfreien universalen Diskurse von Konsensus-Theoretikern wie Peirce, Habermas und Apel. ${ }^{23}$

Auch wenn man hinsichtlich der jüngsten Metadiskurse nicht so weit gehen möchte wie Lyotard, läßt sich der Kern seines Befundes gleichwohl auch wissenssoziologisch stützen und ausfalten. Lyotards Zentralkategorien Delegitimierung und Dissemination lassen sich nämlich in soziologischer Perspektive reformulieren als - so Lübbe - „Kompetenzverlust des Common sense ${ }^{\text {“24 }}$ und „Dispersion des Wissens“ ${ }^{25}$ Beide Prozesse sind im Zuge der Verwissenschaftlichung der modernen Zivilisation wechselseitig aufeinander bezogen. Für den Typus der modernen westlichen Zivilisation, der auch als „Expertengesellschaft ${ }^{\text {‘26 }}$ bzw. als „Wissensgesell-

20 Vgl. hierzu auch Frank 1984, S. 106 ff.; an anderer Stelle hat Lyotard sich so zur Idee des Streites geäußert: „Der Begriff des Streits bezeichnet eine ontologische Situation des richterlichen Urteilens. Richterlich, insofern der Richter angesichts der von jeder Partei vorgebrachten Beweisfuihrung nicht entscheiden kann, denn er verfügt über keine Regel, die für beide Fälle anwendbar wäre. D.h., sowohl die Beweisführung des Klägers [...], als auch die Beweisführung des Verteidigers [...] ist legitim, allerdings in einer anderen Ordnung, in heterogenen Ordnungen." (Lyotard 1985, S. 40).

21 Lyotard 1985, S. 40.

22 Lyotard 1985, S. 33.

23 Vgl. Frank 1984, S. 108-112.

24 Lübbe 1990, S. 83

25 Pfiffner/Stadelmann 1994, S. 146

26 Vgl. hierzu Hitzler 1994, S. 16 


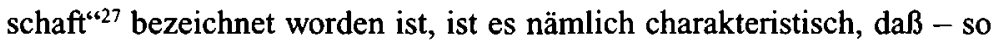
noch einmal Lübbe - „die Annahmen über die Wirklichkeit, wie wir sie stets unseren Handlungen und den ihnen vorausgehenden Entscheidungen zugrunde zu legen haben, nicht mehr unserer Gemeinerfahrung entstammen, vielmehr Produkte ausspezialisierter Erkennntnispraxis, näherhin Hervorbringungen institutionell verselbständigter Wissenschaftspraxis sind." 28

Im Anschluß an Alfred Schütz und Thomas Luckmann, die bereits früh auf die skizzierte - für die westliche Zivilisation charakteristische Entwicklungsdynamik der Verwissenschaftlichung ihrer Gesellschaften hingewiesen haben, ließe sich also folgender Befund festhalten:

Je mehr sich das Allgemeinwissen in verschiedene Versionen aufspaltet, je arbeitsteiliger, je differenzierter eine Gesellschaft ist, desto stärker bilden sich verschiedene Gebiete mit abgegrenztem Sonderwissen aus, das nur noch Spezialisten zugänglich ist. ${ }^{29}$ Dieser Prozeß der Verwissenschaftlichung hat nun für das Problem des Verhältnisses von Wissenschaft und Öffentlichkeit einige bedeutsame Konsequenzen:

(1) Zuerst einmal die, daß sich die Reichweite der verschiedenen Spezialisierungen verkleinert, so daß die Zusammenhänge zwischen den Spezialgebieten nicht nur für Laien, sondern auch für die Experten selbst aus dem Blick geraten. Selbst „Expertenschaft bezieht sich“ - wie Hitzler formuliert - ,nur noch auf Teilbereiche von Sonderwissensgebieten. ${ }^{\text {“30 Wir }}$ können hier von einer Modularisierung des Wissens sprechen, die durchaus auch für die disziplinäre Entwicklung der Linguistik charakteristisch ist: einem kognitivistischen Syntaxtheoretiker dürfte die historische Lexikographie ebenso fremd sein wie die linguistische Informatik einem Neurolinguisten.

(2) Hieraus ergibt sich unmittelbar eine zweite Konsequenz: für den je Einzelnen folgt aus der zentrifugalen Ausdifferenzierung von Sonderwissensbeständen ${ }^{31}$, daß - wie Lübbe formuliert - der „Bereich autarker Urteilszuständigkeiten " 32 fortschreitend sinkt, während der Umfang der an Experten abgetretenen Entscheidungen ständig wächst. Das für die vielfältigen sozialen und persönlichen Probleme relevante Wissen wandert aus dem Bereich des individuell zugänglichen Common sense in den Sektor

\footnotetext{
Vgl. Stehr 1992, S. 107-156; ebenso Walter 1994, S. 269.

Lübke 1990, S. 77.

Vgl. Schütz/Luckmann 1975, S. 113 ff.; vgl. ebenso Bonfadelli 1994, S. 82.

30 Hitzler 1994, S. 22; vgl. ebenso: „Die jeweiligen 'Experten' [...] beschränken sich auf die Bewältigung abgegrenzter Handlungsprobleme und überblicken auch das je eigene Sonderwissensgebiet nicht mehr." (Hitzler 1994, S. 22). Vgl. Pfiffner/Stadelmann 1994, S. 146. Lübbe 1990, S. 82.
}

31

32 
des modularisierten Spezialwissens ab und überantwortet so den Common sense der Bedeutungslosigkeit. Insofern kann mit Recht von einem „Kompetenzverlust des Common sense" (Lübbe) gesprochen werden.

(3) Das Wissen wird aber nicht nur modularisiert, es wird auch in immer rascheren Zyklen erneuert, so daß sich seine Halbwertszeit in immer rasanterem Tempo verkürzt.

(4) Mit der Diversifikation des Wissens, der Beschleunigung der Wissenserneuerung und mit dem Niedergang des Common sense ergibt sich als vierte Konsequenz die exponentiell wachsende Angewiesenheit der Wissensgesellschaft auf das Expertenurteil. ${ }^{33}$ Deshalb entscheiden in weiten Bereichen der Gesellschaft de facto ,(relativ) klar formal definierte Personengruppen verbindlich über mannigfache Probleme nicht nur des sozialen, sondern auch des persönlichen Lebens. “" ${ }^{34}$

(5) Schließlich - und hierin können wir eine fünfte Konsequenz sehen - entsteht aus dem Abwandern entscheidungsrelevanten Wissens in die modularisierten Spezialwissensbestände mit relativ kurzer Haltbarkeitsdauer ein gewaltiger Kommunikationsbedarf zwischen der Commonsense-Welt der Laien, zu der - und dies ist von eminenter Wichtigkeit größtenteils auch die politischen Entscheidungsträger gehören, und der Expertenkultur; ein Kommunikationsbedarf, der um so größer ist, als auch zwischen den Sonderwissensbereichen der Experten immer höhere semantische Barrieren aufwachsen.

Das Verhältnis von Wissenschaft und Öffentlichkeit ist insofern also kein genuines Problem der Sprachwissenschaft, sondern es ist die Konsequenz einer allgemeinen Entwicklungslogik der Wissenschaften, die die Deregulierung der großen Legitimationserzählungen mit einer so eminenten Zerstreuung des Wissens verknüpft, daß die Wissensexperten hinsichtlich ihrer kommunikativen Pflichten gegenüber der Laienwelt in Öffentlichkeit und Politik offenbar rettungslos überfordert sind. Dies um so mehr, als selbst im Binnendiskurs der Experten ein Verlust an Konsensfähigkeit im Hinblick auf bestehende disziplinäre Problemfragen die Regel geworden ist. Die Konsequenzen für das Ansehen der Wissenschaft in der Sphäre der Laienöffentlichkeit liegen - wie Lübbe gezeigt hat - auf der Hand:

„Ist konsistentes, einhelliges Expertenwissen, auf das wir uns doch verlassen können müßten, nicht mehr beschaffbar, widersprechen sich also die Experten ihrerseits [...], so ist Urteilsdistanznahme die durchaus rationale Reaktion der Laienöffentlichkeit $[\ldots]$. “35

33 Vgl. Lübbe 1990, S. 82

34 Hitzler 1994, S. 16.

35 Lübbe 1990, S. 83. 
Nirgendwo ließ sich in der letzten Zeit genau dieser Prozeß deutlicher beobachten als in der öffentlichen Debatte der Rechtschreibreform, die weniger als eine in den Leserbriefspalten sich ausbreitende „LinguistenBeschimpfung ${ }^{636}$ durch Laien, sondern vielmehr als eine immer heftiger werdende öffentliche Auseinandersetzung zwischen linguistischen Experten mit allen fatalen Folgen für das Laien-Publikum angesehen werden muß. Münch hat in seinem bereits zitierten Buch darauf hingewiesen, daß das wachsende "Tempo der Dissenzerzeugung", die Negation einer Expertenmeinung durch die nächste, zwar kein Problem für den Wissenschaftsdiskurs werden muß, daß durch sie jedoch die öffentliche Meinung ins Taumeln gerät: „Es macht sich Ratlosigkeit breit, die zur Suche nach neuen Sicherheiten jenseits der Wissenschaft in Naturheilkunde, neuen Religionen und moralischen Fundamentalismus motiviert." ${ }^{\text {"37 }}$

Auch die Rechtschreibdebatte dürfte in der Öffentlichkeit jene Ratlosigkeit ausgelöst haben, die einerseits Aggression, einen Aufstand der Laien, andererseits aber auch die Sehnsucht nach den einfachen und fundamentalistischen Wahrheiten der "Laien-Linguistik“ hervorruft. Angesichts der Uneinigkeit der Experten gehen die Laien auf Distanz zu den in der selben Sache auseinanderklaffenden Urteilen der Experten. Je divergenter die disziplinären Meinungen, desto offener wird der Markt für jene Laien-Prediger, die vor allem eines verkaufen: den Schein der Wiederherstellung des Common sense sowie die Verabschiedung des zugleich delegitimierten und undurchschaubaren Expertenwissens. Der öffentliche Beifall ist dem sicher, der mit einfachen und verständlichen (aber nicht notwendigerweise wahren) Botschaften die streitenden Experten aus dem Tempel des Wissens jagt.

\section{Wie öffentlichkeitsfähig ist das linguistische Wissen?}

Ich habe bisher zu zeigen versucht, daß die auf den Topos der „Resonanzlosigkeit im öffentlichen Raum " gebrachte Selbstdiagnose der Sprachwissenschaft in nicht unerheblichem Maße als Ausdruck einer Problemlage angesehen werden muß, die einerseits durch den Prozeß der Verwissenschaftlichung der westlichen Zivilisation und andererseits durch die spezifische Dynamik der Kommunikationsgesellschaft geprägt ist. Abschließend möchte ich nun dafür argumentieren, daß es neben den skizzierten strukturellen Rahmenbedingungen für den Resonanzverlust der Linguistik in der Öffentlichkeit auch gleichsam hausgemacht disziplinäre Gründe gibt. Mir geht es dabei nicht etwa darum, die unbestritten großen wissenschaftlichen Leistungen des Faches in den letzten Jahrzehnten in irgend-

$36 \mathrm{Vgl}$. Antos 1996, S. $238 \mathrm{ff}$..

37 Münch 1995, S. 122. 
einer Form hinsichtlich ihres disziplinären Stellenwertes in Zweifel zu ziehen. Die germanistische Linguistik hat auch in ihrer jüngeren Geschichte seit Ende der sechziger Jahre bis in die jüngste Zeit große theoretische Leistungen hervorgebracht. Gleichwohl möchte ich die Frage stellen, ob das Fach nicht gegenwärtig auch deshalb unter einem Verlust an öffentlicher Relevanzerwartung leidet, weil es in wachsendem Maße dazu tendiert, hinsichtlich der fundamentalen Wandlungsprozesse unserer kommunikativen Kultur sowohl seine Fähigkeit zur Identifikation von Problemen als auch das entsprechende Vermögen der Problemlösung zu verlieren, ja - ob es nicht diese Fähigkeit vielleicht sogar bewußt auf dem Altar eines "geisteswissenschaftlichen" Autonomiedenkens opfert.

\subsection{Droht eine „Verzwergung“ der Sprachwissenschaft?}

Es scheint fast so, als indiziere der von Antos konstatierte expandierende Markt der „Laien-Linguistik“ nicht nur die kampflose Preisgabe des „tertiären Sektors" durch die disziplinäre Sprachwissenschaft, sondern darüber hinaus einen tieferliegenden Umstand: das Auseinandertreten nämlich der Relevanzkriterien für die Wahrnehmung und Identifikation lösungsbedürftiger Probleme in der Sprachwissenschaft einerseits und im politischen System sowie in der Öffentlichkeit andererseits. Die Sprachwissenschaft hält offenbar hinter dem Schutzschild des Autonomiearguments gänzlich andere Sachverhalte für wissenschaftlich klärungsbedürftig und klärungsfähig als das politische System und die Öffentlichkeit, ja sie wehrt - mit dem Hinweis auf vorschnelle Verwertungserwartungen das Aufgreifen von Problemen, die im Kontext des politisch-ökonomischen Systems für lösungsbedürftig gehalten werden könnten, weithin und aus grundsätzlichen Erwägungen ab. Es scheint sich deshalb zunehmend - und hier haben wir es mit einer Tendenz zu tun, die auch für andere Geisteswissenschaften charakteristisch ist - eine Problemwahrnehmungsdifferenz aufzutun zwischen den Geisteswissenschaften und ihrer Autonomieinsistenz auf der einen und dem Bereich politisch-ökonomischen Handelns mit seinen Erwartungen an die Diagnose- und Therapiefähigkeit der Wissenschaften auf der anderen Seite. Ohne Zweifel entwickelt sich hier kurz- und mittelfristig eine eminente Marginalisierungsgefahr für jene Geisteswissenschaften, die prinzipiell die mögliche Verwertbarkeit und Praxisrelevanz wissenschaftlichen Wissens mit einer Gefährdung der für Wissenschaft konstitutiven Autonomie der Erkenntnis gleichsetzen.

Eine solche Position ist erst einmal deshalb außerordentlich problematisch, weil sie letztlich der verfehlten Zwei-Kulturen-Hypothese Snows geschuldet ist, derzufolge - wie die Denkschrift „Geisteswissenschaften heute" von Frühwald u. a. formuliert - Kultur ,,als Komplement oder Kompensation andernorts ablaufender Modernisierungsprozesse und der diese betreibenden Wissenschaften angesehen und den Geisteswissenschaften 
[...] zugewiesen" ${ }^{\text {"38 }}$ werde. Wenn sich nämlich die Sprachwissenschaft wie einige andere Geisteswissenschaften auch - mit ihren Erkenntnisgegenständen und ihren Erkenntnisinteressen auf die Seite einer „Kultur“ schlägt, mit der nicht - um mit Mittelstraß zu reden - „,das kulturelle Ganze“, d. h. die „Kultur als Inbegriff der menschlichen Arbeiten und Lebensformen, naturwissenschaftliche und andere Entwicklungen eingeschlossen ${ }^{\text {“39 }}$ gemeint ist, sondern Kultur der Inbegriff des schlechthin anderen von Natur und Technik wird, dann fällt sie jenem Prozeß anheim, den die „Denkschrift" als „Verzwergung der Geisteswissenschaften ${ }^{\prime 440}$ charakterisiert hat. Dann insistiert sie - wie man mit Mittelstraß formulieren könnte - darauf, daß ihr Geschäft das alte bleibt, ,während ihre disziplinären Wettbewerber auf der anderen Kulturseite auf immer neue Weise das Neue besetzen. “41

Zudem verbirgt sich hinter der Zwei-Kulturen-Theorie Snows und des durch sie legitimierten Ausschlusses der technisch-naturwissenschaftlichen Momente des „kulturellen Ganzen“ aus dem disziplinären Erkenntnishorizont der (germanistischen) Sprachwissenschaft letztlich jene cartesianische Zwei-Substanzen-Lehre, die als zuverlässige Grenzmarkierung die Wissenschaften des Geistes vom Reich der Natur- und Technikwissenschaften abzuschirmen versprach. Diese aus dem Wissenschaftssystem des 19. Jahrhunderts stammende Demarkationslinie - und die aus ihr resultierende Polarisierung der Wissenschaften - bestimmt noch immer als sedimentierte Wissenschaftsideologie das Selbstverständnis vieler Geisteswissenschaftler - insbesondere auch im Bereich der Sprachwissenschaft. Unabhängig davon, ob das Erkenntnisobjekt „Geist" mit dem Anspruch auf eigene, verstehende Erkenntnisverfahren gegen "Natur" und ihre Erklärung abgegrenzt wird, oder ob der „Geist“ zur „Natur“ erklärt und als allein den Erkenntnisformen der „Sciences“ zugänglich postuliert wird - beiden Positionen liegt die obsolet gewordene cartesianische Entgegensetzung von Natur und Geist zugrunde, ${ }^{42}$ die durch Charles Percy Snows These von den ,zwei Kulturen ${ }^{443}$ nur noch einmal aktualisiert worden ist. Wie auch immer jedoch die Geisteswissenschaften ihre Selbstbegründung künftig betreiben wollen, die Snowsche Unterscheidung von literarisch-sprachlicher und naturwissenschaftlicher Intelligenz wird als Orientierung kaum mehr taugen, zumal dann nicht, wenn wir es mit Er-

\footnotetext{
Frühwald u.a. 1991, S. 40.

Frühwald u.a. 1991, S. 41.

Frühwald u.a. 1991, S. 40.

41 Frühwald u.a. 1991, S. 33.

$42 \mathrm{Vgl}$. hierzu die Kritik Searles sowohl an der dualistischen als auch an der monistischen Position. In: Searle 1993, etwa S. 16 ff.

43 Vgl. Snow 1967; vgl. ebenso Kreuzer 1987.
} 
kenntnisgegenständen zu tun haben, die - wie die Sprache - weder nur Artefakt des menschlichen Geistes, noch allein Element der natürlichen Ausstattung des Menschen darstellen.

Ein weiterer Umstand spricht dafür, daß die (germanistische) Sprachwissenschaft vielleicht da ihre Position wird überdenken müssen, wo ihr geisteswissenschaftliches Autonomie-Denken das Verhältnis zu Politik und Öffentlichkeit erschwert. Auch die Geisteswissenschaften können sich nämlich nicht länger der Einsicht entziehen, daß - wie Weingart gezeigt hat - mit dem Prozeß der Verwissenschaftlichung der westlichen Gesellschaften ein Funktionswandel des öffentlichen Handelns einhergegangen ist, ${ }^{44} \mathrm{der}$ inzwischen längst einen prinzipiellen Wandel des Verhältnisses von Wissenschaft und politischem System - und damit auch von Wissenschaft und Öffentlichkeit - herbeigeführt hat. Öffentliches Handeln kann sich heute nicht mehr - wie noch im liberalen Staat des 19. Jahrhunderts - darauf beschränken, lediglich ordnend und reaktiv ,Störungen einer sonst als primär selbstregulierend betrachteten ökonomischen und gesellschaftlichen Ordnung " zu beseitigen: Stattdessen ist - so Weingart - „der moderne Staat [...] durch gestaltendes Handeln charakterisiert. Die notwendigen aktiven Interventionen sind per definitionem zukunftsorientiert und komplex. Isolierte ad-hoc-Maßnahmen werden zunehmend unzureichend. [...] Damit ist gesagt, daß gestaltendes öffentliches Handeln sich typischerweise in Planung vollzieht." ${ }^{\text {"4s }}$ Der hier skizzierte - für moderne Wissensgesellschaften charakteristische - Typus öffentlichen Handelns kann also ohne die Nutzung systematischen Wissens, wie es die Einzelwissenschaften bereitstellen, gar nicht mehr auskommen. Es ist deshalb längst eine zentrale Funktion von Wissenschaft geworden, Problemidentifikations- und Problemlösungspotentiale für die Steuerung gesellschaftlicher Prozesse zur Verfügung zu stellen. Durch eine solche Inanspruchnahme systematischen, von den Wissenschaften bereitgestellten Wissens ist die Autonomie der Wissenschaft keineswegs notwendigerweise gefährdet, und zwar deshalb nicht, weil sich das politische System bei der Nutzung wissenschaftlicher Erkenntnis für die Lösung von Problemen nur auf Probleme beziehen kann, die die Wissenschaften zuvor als solche identifiziert haben:

„Probleme werden erst in dem Augenblick zum Gegenstand von Problemlösungsstrategien, in dem die Manipulierbarkeit ihrer Ursachen offenkundig wird, d.h. in dem die Machbarkeit bzw. Veränderbarkeit der Verhältnisse erkannt wird, die sie repräsentieren. Diese Erkenntnis ist zweifellos von einer „Vorleistung" der Wissenschaft im Rahmen ihrer

44 Vgl. Weingart 1976, S. 169

$45 \mathrm{Vgl}$. Weingart 1976, S. 169. 
nach internen Regulativen bestimmten Entwicklung abhängig. Auf diese Weise wird Wissenschaft konstitutiv für die Definition praktischer Probleme." 46

In den Horizont der Verwertung tritt eine Wissenschaft also ohnehin nur unter der Voraussetzung ein, daß es Probleme gibt, die mit ihren kategorialen Mitteln als solche definiert und damit prinzipiell in den Raum möglicher Lösbarkeit versetzt wurden. Insofern ist die verwertungskritische Distanz einer Wissenschaft weniger Ausdruck ihrer „Autonomie“, als vielmehr ihrer Nicht-Partizipation an der Definition von gesellschaftlichen Problemen, deren Lösung prinzipiell in der Reichweite ihrer disziplinären Mittel läge - und in nicht ganz illegitimer Weise würde es diese Problemabstinenz sein, die das Bild einer solchen Wissenschaft in der Perspektive von Politik und Öffentlichkeit bestimmt. Wenn eine Disziplin und das gilt natürlich auch für geisteswissenschaftliche Disziplinen eine ihrer wesentlichen Funktionen, ihre Diagnosefähigkeit, das heißt ihre Fähigkeit, Probleme der Kultur zu identifizieren und sie damit letztlich politischen Lösungsprogrammen zuzuführen, nicht aktiviert, ja diese disziplinäre Reserviertheit als Ausdruck ihrer fachlichen „Autonomie“ legitimiert, dann trägt sie selbst mit dazu bei, daß sie aus der Perspektive des politisch-ökonomischen Systems bzw. der Öffentlichkeit einen Relevanzverlust erleidet. Sie erleidet dann diese Ansehenseinbuße und Resonanzlosigkeit im öffentlichen Raum, die mittelfristig auch mit dem Entzug bzw. der Einschränkung staatlicher Mittelzuweisungen verbunden ist, unabhängig davon, wie glanzvoll und wissenschaftlich eindrucksvoll ihre wissenschaftlichen Leistungen ansonsten sind.

\subsection{Die mangelnde Zeitgenossenschaft der Sprachwissenschaft}

Wenn man von der Resonanzlosigkeit der (germanistischen) Sprachwissenschaft im öffentlichen Raum und von einer schwindenden Öffentlichkeitsfähigkeit linguistischen Wissens sprechen kann, so verdankt sich dieser Befund - so möchte ich meine bisherigen Überlegungen zusammenfassen - drei Ursachen:

(1) Der unzulänglichen externen Kommunikation der Disziplin mit der Öffentlichkeit und dem politisch-ökonomischen System,

(2) der allgemeinen Wissenschaftsentwicklung im Kontext der Entstehung von ,postmodernen" Expertengesellschaften und der hiermit verknüpften Dispersion des Wissens sowie

(3) in nicht unwesentlicher Hinsicht einer Idee von Wissenschaftsautonomie, die ihrerseits von zwei Quellen gespeist wird: (1) von einer Verwertungsskepsis hinsichtlich der Nutzung wissenschaftlichen Wissens,

46 Weingart 1976, S. 216. 
die einhergeht mit der Überzeugung, daß die Verschränkung von Wissenschaft und öffentlich-politischem Handeln für den Bereich der Geistesund Kulturwissenschaften weder relevant, noch ethisch zu rechtfertigen sei und (2) von einer an Descartes und Snow ${ }^{47}$ orientierten Idee der „zwei Kulturen", derzufolge es die Geistes- und Kulturwissenschaften - und auch die Sprachwissenschaft - mit einer Sphäre der Erkenntnis zu tun haben - im Falle der Sprachwissenschaft mit der Sphäre der sprachlichzeichenhaften Hervorbringungen des menschlichen Geistes -, in der sich Fragen der technischen Kultur und ihrer Transformationen und damit Fragen der technologischen Wandlungsprozesse unserer kommunikativen Umwelt und der hierdurch verursachten Folgen nicht stellen.

Natürlich ist diese Feststellung eine überpointierte - und eigentlich unzulässig generalisierende - Charakterisierung unserer Disziplin, die dem komplexen und differenzierten Leistungsspektrum des Faches insgesamt sicher nicht gerecht werden kann. Denn selbstverständlich haben - um nur einige Beispiele zu nennen - etwa die Arbeiten von Stötzel/Wengeler, von Strauß/Haß/Harras zum öffentlichen Sprachgebrauch und seiner jüngeren Geschichte oder von Heringer zum Zusammenhang von Politik, Sprache und Moral gerade in der sprachkritischen Öffentlichkeit große Aufmerksamkeit erfahren und natürlich fördern sprachhistorische Unternehmungen wie die Sprachgeschichte von von Polenz und das Frühneuhochdeutsche Wörterbuch von Goebel/Reichmann, grammatische Grundlagenarbeiten wie Eisenbergs Grammatik und Heringers dependentielle Syntax oder das geradezu enzyklopädeische Unternehmen der von Steger und Wiegand herausgegebenen Handbücher für Sprach- und Kommunikationswissenschaft das disziplinäre Ansehen der Sprachwissenschaft.

Gleichwohl gibt es - trotz dieser eindrucksvollen Hervorbringungen des Faches - Anlaß zu der Feststellung, daß der Ansehensverlust der Sprachwissenschaft im öffentlichen Raum nicht nur durch Marketingfehler oder allgemeine Randbedingungen der Wissenschaftsentwicklung, sondern auch durch den disziplinären Wissenschaftsprozeß selber mitbewirkt wurde - nicht durch die fachlichen Monumente, die er hervorgebracht hat, sondern vielmehr durch die, deren offenkundige Abwesenheit immer schmerzlicher bewußt wird. Ich möchte hierzu abschließend einige Anmerkungen machen.

Rainer Wimmer hat in dem bereits zitierten Statement zu der Frage, ob es eine interessierte Öffentlichkeit für die germanistische Linguistik gebe, mit Recht darauf hingewiesen, daß es trotz der Modernisierungsschübe, die das Fach in den sechziger und siebziger Jahren erfahren habe, bis heute

47 Snow setzt sich allerdings kritisch mit der geisteswissenschaftlichen Halbierung des Kulturbegriffs auseinander (vgl. Snow 1967, S. 14, S. 20 f.). 
nicht gelungen sei, „Problemfelder, die sich aus den Anwendungsgebieten in Abhängigkeit von neuen gesellschaftlichen Entwicklungen geradezu aufdrängen, in ausreichendem Maße zu bearbeiten und die Arbeitsergebnisse in die Praxis hinein zu vermitteln." $\mathrm{Zu}$ diesen Problemfeldern zählen für ihn „der Einfluß der neuen Medien auf den Umgang mit Sprache und mit sprachlichen Fähigkeiten und Fertigkeiten [...], der Einfluß des Fernsehens und anderer Kommunikationsmedien auf die Sprachentwicklung der Gegenwart, Veränderungen des öffentlichen Sprachgebrauchs (insbesondere im Bereich Sprache und Politik)“" sowie „Sprachpolitik in Europa". Diese Problemfelder seien - so Wimmer - durchaus sporadisch in Angriff genommen worden, es mangele allerdings an kontinuierlichen Forschungsprogrammen, die effektvolle Hilfen für die Praxis bereitstellen könnten. ${ }^{48}$ In der Tat ist es auffällig, wie wenig die germanistische Sprachwissenschaft - im Gegensatz zur germanistischen Literaturwissenschaft und zu anderen Philologien - bislang produktiv auf die Anregungen der Denkschrift „Geisteswissenschaften heute" von Frühwald u. a. eingegangen ist, in der die Autoren 1991 die Geisteswissenschaften aufforderten, dadurch einen Beitrag zur Selbstverständigung der modernen Industriegesellschaft zu leisten, „daß sie 'als interdisziplinäre Kulturwissenschaft mit offenen Augen für die Geschichte der technischen Welt' (Böhme 1989, S. 30) eine kulturwissenschaftlich orientierte Medienwissenschaft ausbilden“, die - so die Autoren - „die technisch vermittelte Massenkultur der modernen Industriegesellschaft $\mathrm{zu}$ ihrem Gegenstand ${ }^{\prime 49}$ machte. Obgleich Sprache und Kommunikation naturgemäß im genuinen Zentrum linguistischer Erkenntnisbemühungen stehen, scheinen die durch technische Medien organisierten Bedingungen der Prozessierung von Sprache und die durch sie hervorgerufenen grundlegenden Wandlungsprozesse der kommunikativen Umwelt das Interesse der Sprachwissenschaft bislang kaum gefunden zu haben.

Vielleicht darf man einen Grund hierfür in dem Umstand suchen, daß die germanistische Sprachwissenschaft den theoretischen Diskurs darüber, was das Erkenntnisobjekt der Sprachwissenschaft sei, weithin einem linguistischen Kognitivismus überlassen hat, der alle Bereiche der historischen und sozialen Erscheinung der Sprache zum Epiphänomen erklärt und somit auch die historisch variierenden medialen Bedingungen der Prozessierung von Sprache theoretisch diskreditiert hat.

Die stumme Distanz der germanistischen Sprachwissenschaft zu dem in den Geisteswissenschaften mit zunehmender Intensität geführten medientheoretischen Diskurs, der über die beteiligten Wissenschaften hinaus eine

48 Vgl. Wimmer 1994, S. 54.

49 Frühwald u. a. 1991, S. 156. 
große Aufmerksamkeit in Öffentlichkeit und Politik findet, ist aber nur ein Symptom eines gravierenderen Abseitsstehens: während - wiederum mitangeregt durch die Frühwald-Denkschrift - in den nicht-germanistischen Sprach- und Literaturwissenschaften und in der Geschichtswissenschaft eine intensive Debatte über eine Neubestimmung der Geisteswissenschaften als Kulturwissenschaften eingesetzt hat ${ }^{50}$,

in der diesen die Aufgabe zugedacht wird, ,als Instrument interkultureller Bildung und anthropologischer Erkenntnis einen genuinen Beitrag zum Problem der Reintegration der technologischen Zivilisation in die gesellschaftliche Kultur der Zukunft zu leisten"sl, sind Ansätze einer Beteiligung der germanistischen Sprachwissenschaft an dieser Diskussion einer möglichen kulturwissenschaftlichen Erneuerung der Geisteswissenschaften - so weit ich sehe - nicht zu erkennen. Die Nicht-Beteiligung der Sprachwissenschaft ist um so befremdlicher, als im Zentrum dieser Debatte ein in der Tradition Herders und Cassirers konzeptionalisierter, sprachlich fundierter Kulturbegriff steht, der die diskursive Abwesenheit der Sprachwissenschaft einigermaßen unverständlich erscheinen läßt:

„Die sprachliche Fundierung aller kulturellen Schöpfungen [...] geht in die Analyse der gesamten Symbol- und Zeichenwelt über [...], ohne die keine menschlicheSelbstorganisation möglich ist [...]. Dieser Kulturbegriff deckt sowohl alle Text- und Schriftwissenschaften ab als auch den Überschritt zu den allgemeinen Kommunikationsleistungen. Sowohl die Beziehung der schriftlichen Sprache zur gesprochenen Sprache und zur Sprache schlechthin wird vom Kulturbegriff umfaßt als auch die Ausweitung auf alle modernen Kommunikationsleistungen im audiovisuellen Bereich." 52

Gerade in der Partizipation an den für sie einschlägigen kultursemiotischen Fragestellungen, mit denen die Sprachwissenschaft zur Hochzeit des Strukturalismus interdisziplinäre Strahlkraft für das Projekt einer zeichentheoretischen Begründung der Geisteswissenschaften gewonnen hatte, sowie in der Beteiligung an den kultur- und medienwissenschaftlichen Innovationsbemühungen benachbarter Geisteswissenschaften könnte die Sprachwissenschaft vielleicht ihre öffentliche Resonanz wieder erhöhen und diese Resonanz auch für die disziplinären Bereiche fruchtbar machen, deren Praxisrelevanz schwieriger zu vermitteln ist.

In jedem Fall ist es hohe Zeit, daß sich die Sprachwissenschaft aus ihrer selbst gewählten kultur- und medienwissenschaftlichen Isolation befreit und so - neben den bereits skizzierten Gründen - den gegenwärtig gewichtigsten Anlaß für die Beschädigung ihres Verhältnisses zur Öffentlichkeit aus dem Wege räumt. Auch die professionellste Optimierung ihres

so Frühwald u.a. 1991, S. 11.

51 Frühwald, u. a. 1991, S. 11.

52 Frühwald u.a. 1991, S. 139. 
Marketings wird nicht überspielen können, daß sie an dem interdisziplinären Diskurs zur kulturwissenschaftlichen Erneuerung der Geisteswissenschaften bislang nicht beteiligt ist.

\section{Literatur}

Antos, G. 1996: Laien-Linguistik. Studien zu Sprach- und Kommunikationsproblemen im Alltag. Am Beispiel von Sprachratgebern und Kommunikationstrainings. Tübingen.

Antos, G. o. J.: Linguistik in der Öffentlichkeit. Rezeption und Rezeptionsverweigerung linguistischen Wissens durch die Öffentlichkeit am Beispiel von Sprachratgebern und Kommunikationstrainings. DFG-Projektskizze. [Stand August 1998].

Bachorsky, H. J. 1994: Überlegungen zum Leitthema: Die Germanistik und die Öffentlichkeit. In: Mitteilungen des Deutschen Germanistenverbandes, Heft 3, September 1994, S. 10-15.

Böhme, H./ Scherpe, K. R. (Hg.) 1996: Literatur- und Kulturwissenschaften. Positionen, Theorien, Modelle. Hamburg.

Böke, K./ Jung, M./ Wengeler, M. (Hg.) 1996: Öffentlicher Sprachgebrauch. Praktische, theoretische und historische Perspektiven. Opladen.

Bonfadelli, H. 1994: Die Wissenskluft-Perspektive. Massenmedien und gesellschaftliche Information. Konstanz.

Cerquiglini, B./ Gumbrecht, H.U.(Hg.) 1983: Der Diskurs der Literatur- und Sprachhistorie. Wissenschaftsgeschichte als Innovationsvorgabe. Frankfurt a. M.

Frank, M. 1984: Was ist Neostrukturalismus. Frankfurt a. M.

Frühwald, W./ Jauß, H. R./ Koselleck, R./ Mittelstraß, J./ Steinwachs, B. 1991: Geisteswissenschaften heute. Eine Denkschrift. Frankfurt a. M.

Gumbrecht, H. U./ Pfeiffer, L. K. 1988: Materialität der Kommunikation. Frankfurt a. M.

Hitzler, R. 1994: Wissen und Wesen des Experten. In: Hitzler/Honer/Maeder 1994.

Hitzler, R./ Honer, A./ Maeder, Chr. (Hg.) 1994: Expertenwissen. Opladen.

Hoberg, R. 1997: Öffentlichkeit und Sprachwissenschaft. In: Muttersprache 107, S. 54-63.

Jäger, L. 1996: Expertenkultur und Sprachkultur. „Innersprachliche Mehrsprachigkeit" und das Problem der Transparenz des Expertenwissens. In: Böke/ Jung/ Wengeler (Hg.) 1996, S. 66-76.

Klein, E./ Pouradier Duteil, F./ Wagner, H.W. 1990: Betriebslinguistik und Linguistikbetrieb. Akten des 24. Linguistischen Kolloquiums. Universität Bremen, 4.-6. September 1989. Tübingen.

Kreuzer, H. (Hg.) 1987: Die zwei Kulturen. Literarische und naturwissenschaftliche Intelligenz. C.P. Snows These in der Diskussion. München.

Kuhn, Th. S. 1973: Die Struktur der wissenschaftlichen Revolution. Frankfurt a. M.

Löw, R./ Koslowski, P./ Spaemann, R. (Hg.) 1990: Expertenwissen und Politik. Weinheim.

Lübbe, H. 1990: Politische Entscheidung und Fachwissen. In: Löw/ Koslowski/ Spaemann 1990, S. 77-90.

Luhmann, N. 1986: Ökologische Kommunikation. Kann die moderne Gesellschaft sich auf ökologische Gefährdungen einstellen? Opladen.

Lyotard, J.F. u. a. 1985: Immaterialität und Postmoderne. Berlin.

Lyotard, J. F. (1979) 1986: Das postmoderne Wissen. Wien. 
Münch, R. 1995: Die Dynamik der Kommunikationsgesellschaft. Frankfurt a.M. Pfiffner, M./ Stadelmann, P. 1994: Expertenwissen und Wissensexperten. In: Hitzler/ Honer/ Maeder (Hg.) 1994.

Schlesier, R. 1996: Das Staunen ist der Anfang der Anthropologie. In: Böhme/ Scherpe 1996, S. 47-59.

Schütz, A./ Luckmann, Th. 1975: Strukturen der Lebenswelt. Neuwied, Darmstadt.

Searle, J.R. 1993: Die Wiederkehr des Geistes. München.

Snow, Ch.P. 1967: Die zwei Kulturen. Literarische und naturwissenschaftliche Intelligenz. Stuttgart.

Stehr, N. 1992: Experts, Counsellors and Advisers. In: Stehr, N./ Ericson, R.V. (eds.) 1992: The Culture as Power of Knowledge. Inquiries into Contemporary Societies. Berlin, New York, S. 107-156.

Walter, W. 1994: Strategien der Politikberatung. Die Interpretation der Sachverständigenrolle im Lichte von Experteninterviews. In: Hitzler/ Honer/ Maeder (Hg.) 1994.

Weingart, P./ Prinz, W./ Kastner, M./ Maasen, S./ Walter, W. 1991: Die sogenannten Geisteswissenschaften: Außenansichten. Die Entwicklung der Geisteswissenschaften in der BRD 1954-1987. Frankfurt a. M.

Wimmer, R. 1994: Interessierte Öffentlichkeit für germanistische Linguistik. In: Mitteilungen des Deutschen Germanistenverbandes, Heft 3, September 1994, S. 51-56.

Winnacker, E.L. 1998: Plädoyer für eine neue Wissenschaftskultur. Grundlagenforschung in Zeiten der Innovationstümelei und Millenomanie. In: Süddeutsche Zeitung Nr. 5, Donnerstag 8. Januar 1998, S. 33. 